PROCEEDINGS OF THE

AMERICAN MATHEMATICAL SOCIETY

Volume 134, Number 10, October 2006, Pages 3071-3079

S 0002-9939(06)08332-8

Article electronically published on May 1, 2006

\title{
THE FIRST EIGENVALUE OF A CLOSED MANIFOLD WITH POSITIVE RICCI CURVATURE
}

\author{
JUN LING \\ (Communicated by Jozef Dodziuk)
}

\begin{abstract}
We give a new estimate on the lower bound for the first positive eigenvalue of the Laplacian on a closed manifold with positive Ricci curvature in terms of the lower bound of the Ricci curvature and the largest interior radius of the nodal domains of eigenfunctions of the eigenvalue.
\end{abstract}

\section{INTRODUCTION}

It has been proved by Li-Yau [5] and Zhong-Yang [12] that if $M^{n}$ is a closed $n$-dimensional manifold with nonnegative Ricci curvature, then the first nonzero eigenvalue $\lambda$ of the manifold has a lower bound

$$
\lambda \geq \frac{\pi^{2}}{d^{2}} .
$$

On the other hand, if the Ricci curvature $\operatorname{Ric}\left(M^{n}\right)$ has a positive lower bound $(n-1) K$ for some positive constant $K$, the Lichnerowicz Theorem states that

$$
\lambda \geq n K .
$$

The Lichnerowicz-type estimate (1.1) is nice and optimal for positive $K$. But it gives no information when the Ricci curvature lower bound is zero. Therefore it is an interesting problem to find a unified lower bound of the first positive eigenvalue $\lambda$ in terms of the lower bound $(n-1) K$ of the Ricci curvature and other geometric quantities for a closed manifold with positive Ricci curvature so that the lower bound of $\lambda$ does not vanish as the lower bound of the Ricci curvature does. P. Li conjectured that the lower bound for the first positive eigenvalue $\lambda$ is $(n-1) K+$ $\pi^{2} / d^{2}$. D. Yang [1] showed that the coefficient before the Ricci lower bound $(n-1) K$ is $1 / 4$. Along the line of the Li Conjecture, we give a new estimate on the lower bound of the first positive eigenvalue of a closed Riemannian manifold with positive lower bound of Ricci curvature in terms of the lower bound of the Ricci curvature and the largest interior radius of the nodal domains of eigenfunctions of the eigenvalue. Instead of using Zhong-Yang's canonical function or the "midrange" of the normalized eigenfunction of the first positive eigenvalue in the proof, we use a function $\xi$ that the author constructed in 8$]$ for the construction of the suitable

Received by the editors October 15, 2004 and, in revised form, April 28, 2005.

2000 Mathematics Subject Classification. Primary 58J50, 35P15; Secondary 53C21.

Key words and phrases. Eigenvalue, lower bound, closed Riemannian manifold.

(C)2006 American Mathematical Society 
test function and use the structure of the nodal domains of the eigenfunction. We have the following result.

Theorem 1.1. Let $M$ be a closed $n$-dimensional Riemannian manifold with Ricci curvature $\operatorname{Ric}(M)$ bounded below by

$$
\operatorname{Ric}(M) \geq(n-1) K>0 .
$$

Then the first positive eigenvalue $\lambda$ of the Laplacian $\Delta$ on $M$ has the lower bound

$$
\lambda \geq \frac{1}{2}(n-1) K+\frac{\pi^{2}}{4 r^{2}},
$$

where $r$ is the largest interior radius of the nodal domains of eigenfunctions of the eigenvalue.

We derive some preliminary estimates and conditions for test functions in the next section and construct the needed test function and prove the main result in the last section.

\section{Preliminary estimates}

The classic Lichnerowicz Theorem [6] states that if $M$ is a closed $n$-dimensional manifold whose Ricci curvature satisfies (1.2), then the first positive eigenvalue $\lambda$ of the Laplacian on $M$ has a lower bound in (1.1). For completeness and consistency, we use gradient estimate in [3- $[5]$ and [10] to derive the Lichnerowicz estimate.

Lemma 2.1 (Lichnerowicz). Under the conditions in Theorem 1.1, estimate (1.1) holds.

Proof. Let $v$ be a normalized eigenfunction of the first positive eigenvalue $\lambda$ of the Laplacian $\Delta$ on $M$ such that

$$
\sup _{M} v=1 \quad \text { and } \quad \inf _{M} v=-k
$$

with $0<k \leq 1$. The function $v$ satisfies the following equation:

$$
\Delta v=-\lambda v \quad \text { in } M .
$$

Take an orthonormal frame $\left\{e_{1}, \ldots, e_{n}\right\}$ of $M$ about $x_{0} \in M$. At $x_{0}$ we have

$$
\nabla_{e_{j}}\left(|\nabla v|^{2}\right)\left(x_{0}\right)=\sum_{i=1}^{n} 2 v_{i} v_{i j}
$$

and

$$
\begin{aligned}
\Delta\left(|\nabla v|^{2}\right)\left(x_{0}\right) & =2 \sum_{i, j=1}^{n} v_{i j} v_{i j}+2 \sum_{i, j=1}^{n} v_{i} v_{i j j} \\
& =2 \sum_{i, j=1}^{n} v_{i j} v_{i j}+2 \sum_{i, j=1}^{n} v_{i} v_{j j i}+2 \sum_{i, j=1}^{n} \mathrm{R}_{i j} v_{i} v_{j} \\
& =2 \sum_{i, j=1}^{n} v_{i j} v_{i j}+2 \nabla v \nabla(\Delta v)+2 \operatorname{Ric}(\nabla v, \nabla v) \\
& \geq 2 \sum_{i=1}^{n} v_{i i}^{2}+2 \nabla v \nabla(\Delta v)+2(n-1) K|\nabla v|^{2} \\
& \geq \frac{2}{n}(\Delta v)^{2}-2 \lambda|\nabla v|^{2}+2(n-1) K|\nabla v|^{2} .
\end{aligned}
$$


Thus at all points $x \in M$,

$$
\frac{1}{2} \Delta\left(|\nabla v|^{2}\right) \geq \frac{1}{n} \lambda^{2} v^{2}+[(n-1) K-\lambda]|\nabla v|^{2} .
$$

On the other hand, after multiplying (2.2) by $v$ and integrating both sides over $M$, we have

$$
\int_{M} \lambda v^{2} d x=-\int_{M} v \Delta v d x=\int_{M}|\nabla v|^{2} d x
$$

Integrating (2.3) over $M$ and using the above equality, we get

$$
0 \geq \int_{M}(n K-\lambda) \frac{n-1}{n} \lambda v^{2} d x
$$

Therefore (1.1) holds.

Lemma 2.2. Let $v$ be, as above, the normalized eigenfunction for the first positive eigenvalue $\lambda$. Then $v$ satisfies the following:

$$
\frac{|\nabla v|^{2}}{b^{2}-v^{2}} \leq \lambda
$$

where $b>1$ is an arbitrary constant.

Proof. Consider the function

$$
P(x)=|\nabla v|^{2}+A v^{2},
$$

where $A=\lambda(1+\epsilon)$ for small $\epsilon>0$. Function $P$ must achieve its maximum at some point $x_{0} \in M$. We claim that $\nabla v\left(x_{0}\right)=0$.

If on the contrary $\nabla v\left(x_{0}\right) \neq 0$, then we can rotate the local orthonormal about $x_{0}$ such that

$$
\left|v_{1}\left(x_{0}\right)\right|=\left|\nabla v\left(x_{0}\right)\right| \neq 0 \quad \text { and } \quad v_{i}\left(x_{0}\right)=0, \quad i \geq 2 .
$$

Since $P$ achieves its maximum at $x_{0}$, we have

$$
\nabla P\left(x_{0}\right)=0 \quad \text { and } \quad \Delta P\left(x_{0}\right) \leq 0 .
$$

That is, at $x_{0}$ we have

$$
\begin{gathered}
0=\frac{1}{2} \nabla_{i} P=\sum_{j=1}^{n} v_{j} v_{j i}+A v v_{i}, \\
v_{11}=-A v \quad \text { and } \quad v_{1 i}=0, \quad i \geq 2,
\end{gathered}
$$

and

$$
\begin{aligned}
0 & \geq \frac{1}{2} \Delta P\left(x_{0}\right)=\sum_{i, j=1}^{n}\left(v_{j i} v_{j i}+v_{j} v_{j i i}+A v_{i} v_{i}+A v v_{i i}\right) \\
& =\sum_{i, j=1}^{n}\left(v_{j i}^{2}+v_{j}\left(v_{i i}\right)_{j}+\mathrm{R}_{j i} v_{j} v_{i}+A v_{i i}^{2}+A v v_{i i}\right) \\
& =\sum_{i, j=1}^{n} v_{j i}^{2}+\nabla v \nabla(\Delta v)+\operatorname{Ric}(\nabla v, \nabla v)+A|\nabla v|^{2}+A v \Delta v \\
& \geq v_{11}^{2}+\nabla v \nabla(\Delta v)+(n-1) K|\nabla v|^{2}+A|\nabla v|^{2}+A v \Delta v \\
& =(-A v)^{2}-\lambda|\nabla v|^{2}+(n-1) K|\nabla v|^{2}+A|\nabla v|^{2}-\lambda A v^{2} \\
& =(A-\lambda+(n-1) K)|\nabla v|^{2}+A v^{2}(A-\lambda),
\end{aligned}
$$


where we have used (2.7) and (1.2). Therefore at $x_{0}$,

$$
0 \geq(A-\lambda)|\nabla v|^{2}+A(A-\lambda) v^{2},
$$

that is,

$$
\left|\nabla v\left(x_{0}\right)\right|^{2}+\lambda(1+\epsilon) v\left(x_{0}\right)^{2} \leq 0 .
$$

Thus $\nabla v\left(x_{0}\right)=0$. This contradicts $\nabla v\left(x_{0}\right) \neq 0$. So the above claim is true.

Therefore we have $\nabla v\left(x_{0}\right)=0$,

$$
P\left(x_{0}\right)=\left|\nabla v\left(x_{0}\right)\right|^{2}+A v\left(x_{0}\right)^{2}=A v\left(x_{0}\right)^{2} \leq A,
$$

and at all $x \in M$

$$
|\nabla v(x)|^{2}+A v(x)^{2}=P(x) \leq P\left(x_{0}\right) \leq A .
$$

Letting $\epsilon \rightarrow 0$ in the above inequality, the estimate (2.5) follows.

We want to improve the upper bound in (2.5) further and proceed in the following way.

Define a function $Z$ on $\left[-\sin ^{-1}(k / b), \sin ^{-1}(1 / b)\right]$ by

$$
Z(t)=\max _{x \in M, t=\sin ^{-1}(v(x) / b)} \frac{|\nabla v|^{2}}{b^{2}-v^{2}} / \lambda .
$$

From (2.5) we have

$$
Z(t) \leq 1 \quad \text { on }\left[-\sin ^{-1}(k / b), \sin ^{-1}(1 / b)\right] .
$$

For convenience, in this paper we let

$$
\alpha=\frac{1}{2}(n-1) K \quad \text { and } \quad \delta=\alpha / \lambda .
$$

By (1.1) we have

$$
\delta \leq \frac{n-1}{2 n} .
$$

We have the following conditions on the test function $Z$.

Theorem 2.3. If the function $z:\left[-\sin ^{-1}(k / b), \sin ^{-1}(1 / b)\right] \mapsto \mathbf{R}^{1}$ satisfies the following:

(1) $z(t) \geq Z(t), \quad t \in\left[-\sin ^{-1}(k / b), \sin ^{-1}(1 / b)\right]$,

(2) there exists some $x_{0} \in M$ such that $z\left(t_{0}\right)=Z\left(t_{0}\right)$ at point $t_{0}=\sin ^{-1}\left(v\left(x_{0}\right) / b\right)$,

(3) $z\left(t_{0}\right)>0$, and

(4) $z^{\prime}\left(t_{0}\right) \sin t_{0} \geq 0$

then we have

$$
0 \leq \frac{1}{2} z^{\prime \prime}\left(t_{0}\right) \cos ^{2} t_{0}-z^{\prime}\left(t_{0}\right) \cos t_{0} \sin t_{0}-z\left(t_{0}\right)+1-2 \delta \cos ^{2} t_{0} .
$$

Proof. Define

$$
J(x)=\left\{\frac{|\nabla v|^{2}}{b^{2}-v^{2}}-\lambda z\right\} \cos ^{2} t,
$$

where $t=\sin ^{-1}(v(x) / b)$. Then

$$
J(x) \leq 0 \quad \text { for } x \in M \quad \text { and } \quad J\left(x_{0}\right)=0 .
$$

If $\nabla v\left(x_{0}\right)=0$, then

$$
0=J\left(x_{0}\right)=-\lambda z \cos ^{2} t
$$


This contradicts condition (3) in the theorem. Therefore

$$
\nabla v\left(x_{0}\right) \neq 0 .
$$

The Maximum Principle implies that

$$
\nabla J\left(x_{0}\right)=0 \quad \text { and } \quad \Delta J\left(x_{0}\right) \leq 0 .
$$

$J(x)$ can be rewritten as

$$
J(x)=\frac{1}{b^{2}}|\nabla v|^{2}-\lambda z \cos ^{2} t .
$$

Thus (2.13) is equivalent to

$$
\left.\frac{2}{b^{2}} \sum_{i} v_{i} v_{i j}\right|_{x_{0}}=\left.\lambda \cos t\left[z^{\prime} \cos t-2 z \sin t\right] t_{j}\right|_{x_{0}}
$$

and

$$
\begin{aligned}
0 \geq & \frac{2}{b^{2}} \sum_{i, j} v_{i j}^{2}+\frac{2}{b^{2}} \sum_{i, j} v_{i} v_{i j j}-\lambda\left(z^{\prime \prime}|\nabla t|^{2}+z^{\prime} \Delta t\right) \cos ^{2} t \\
& +4 \lambda z^{\prime} \cos t \sin t|\nabla t|^{2}-\left.\lambda z \Delta \cos ^{2} t\right|_{x_{0}} .
\end{aligned}
$$

Rotate the frame so that $v_{1}\left(x_{0}\right) \neq 0$ and $v_{i}\left(x_{0}\right)=0$ for $i \geq 2$. Then (2.14) implies

$$
\left.v_{11}\right|_{x_{0}}=\left.\frac{\lambda b}{2}\left(z^{\prime} \cos t-2 z \sin t\right)\right|_{x_{0}} \text { and }\left.\quad v_{1 i}\right|_{x_{0}}=0 \text { for } i \geq 2 \text {. }
$$

Now we have

$$
\begin{aligned}
\left.|\nabla v|^{2}\right|_{x_{0}} & =\left.\lambda b^{2} z \cos ^{2} t\right|_{x_{0}}, \\
\left.|\nabla t|^{2}\right|_{x_{0}} & =\frac{|\nabla v|^{2}}{b^{2}-v^{2}}=\left.\lambda z\right|_{x_{0}}, \\
\left.\frac{\Delta v}{b}\right|_{x_{0}} & =\Delta \sin t=\cos t \Delta t-\left.\sin t|\nabla t|^{2}\right|_{x_{0}}, \\
\left.\Delta t\right|_{x_{0}} & =\frac{1}{\cos t}\left(\sin t|\nabla t|^{2}+\frac{\Delta v}{b}\right) \\
& =\left.\frac{1}{\cos t}\left[\lambda z \sin t-\frac{\lambda}{b} v\right]\right|_{x_{0}}, \quad \text { and } \\
\left.\Delta \cos ^{2} t\right|_{x_{0}} & =\Delta\left(1-\frac{v^{2}}{b^{2}}\right)=-\frac{2}{b^{2}}|\nabla v|^{2}-\frac{2}{b^{2}} v \Delta v \\
& =-2 \lambda z \cos ^{2} t+\left.\frac{2}{b^{2}} \lambda v^{2}\right|_{x_{0}} .
\end{aligned}
$$

Therefore,

$$
\begin{aligned}
& \left.\frac{2}{b^{2}} \sum_{i, j} v_{i j}^{2}\right|_{x_{0}} \geq \frac{2}{b^{2}} v_{11}^{2} \\
& =\frac{\lambda^{2}}{2}\left(z^{\prime}\right)^{2} \cos ^{2} t-2 \lambda^{2} z z^{\prime} \cos t \sin t+\left.2 \lambda^{2} z^{2} \sin ^{2} t\right|_{x_{0}}
\end{aligned}
$$




$$
\begin{aligned}
&\left.\frac{2}{b^{2}} \sum_{i, j} v_{i} v_{i j j}\right|_{x_{0}}=\frac{2}{b^{2}}(\nabla v \nabla(\Delta v)+\operatorname{Ric}(\nabla v, \nabla v)) \\
& \geq \frac{2}{b^{2}}\left(\nabla v \nabla(\Delta v)+(n-1) K|\nabla v|^{2}\right) \\
&=-2 \lambda^{2} z \cos ^{2} t+\left.4 \alpha \lambda z \cos ^{2} t\right|_{x_{0}} \\
&-\left.\lambda\left(z^{\prime \prime}|\nabla t|^{2}+z^{\prime} \Delta t\right) \cos ^{2} t\right|_{x_{0}} \\
&=-\lambda^{2} z z^{\prime \prime} \cos ^{2} t-\lambda^{2} z z^{\prime} \cos t \sin t \\
&+\left.\frac{1}{b} \lambda^{2} z^{\prime} v \cos t\right|_{x_{0}}
\end{aligned}
$$

and

$$
\begin{aligned}
& 4 \lambda z^{\prime} \cos t \sin t|\nabla t|^{2}-\left.\lambda z \Delta \cos ^{2} t\right|_{x_{0}} \\
& =4 \lambda^{2} z z^{\prime} \cos t \sin t+2 \lambda^{2} z^{2} \cos ^{2} t-\left.\frac{2}{b} \lambda^{2} z v \sin t\right|_{x_{0}} .
\end{aligned}
$$

Putting these results into (2.15) we get

$$
\begin{aligned}
0 \geq & -\lambda^{2} z z^{\prime \prime} \cos ^{2} t+\frac{\lambda^{2}}{2}\left(z^{\prime}\right)^{2} \cos ^{2} t+\lambda^{2} z^{\prime} \cos t(z \sin t+\sin t) \\
& +2 \lambda^{2} z^{2}-2 \lambda^{2} z+\left.4 \alpha \lambda z \cos ^{2} t\right|_{x_{0}},
\end{aligned}
$$

where we used (2.16). Now

$$
z\left(t_{0}\right)>0
$$

by condition (3) in the theorem. Dividing two sides of (2.17) by $\left.2 \lambda^{2} z\right|_{x_{0}}$, we have

$$
\begin{aligned}
0 \geq & -\frac{1}{2} z^{\prime \prime}\left(t_{0}\right) \cos ^{2} t_{0}+\frac{1}{2} z^{\prime}\left(t_{0}\right) \cos t_{0}\left(\sin t_{0}+\frac{\sin t_{0}}{z\left(t_{0}\right)}\right)+z\left(t_{0}\right) \\
& -1+2 \delta \cos ^{2} t_{0}+\frac{1}{4 z\left(t_{0}\right)}\left(z^{\prime}\left(t_{0}\right)\right)^{2} \cos ^{2} t_{0} .
\end{aligned}
$$

Therefore,

$$
\begin{aligned}
0 \geq & -\frac{1}{2} z^{\prime \prime}\left(t_{0}\right) \cos ^{2} t_{0}+z^{\prime}\left(t_{0}\right) \cos t_{0} \sin t_{0}+z\left(t_{0}\right)-1+2 \delta \cos ^{2} t_{0} \\
& +\frac{1}{4 z\left(t_{0}\right)}\left(z^{\prime}\left(t_{0}\right)\right)^{2} \cos ^{2} t_{0}+\frac{1}{2} z^{\prime}\left(t_{0}\right) \sin t_{0} \cos t_{0}\left[\frac{1}{z\left(t_{0}\right)}-1\right] .
\end{aligned}
$$

Conditions (1), (2) and (4) in the theorem imply that $0<z\left(t_{0}\right)=Z\left(t_{0}\right) \leq 1$ and $z^{\prime}\left(t_{0}\right) \sin t_{0} \geq 0$. Thus the last two terms in (2.19) are nonnegative and (2.12) follows.

\section{Proof of the main Result}

Proof of Theorem 1.1. Let

$$
z(t)=1+\delta \xi(t)
$$

where $\xi$ is the function defined by (3.9) in Lemma 3.1. We claim that

$$
Z(t) \leq z(t) \quad \text { on }\left[-\sin ^{-1}(k / b), \sin ^{-1}(1 / b)\right] \text {. }
$$


Lemma 3.1 implies that for $t \in\left[-\sin ^{-1}(k / b), \sin ^{-1}(1 / b)\right]$ we have the following:

$$
\begin{aligned}
& \frac{1}{2} z^{\prime \prime} \cos ^{2} t-z^{\prime} \cos t \sin t-z=-1+2 \delta \cos ^{2} t \\
& z^{\prime}(t) \sin t \geq 0 \\
& 0<1-\left(\frac{\pi^{2}}{4}-1\right) \frac{n-1}{2 n} \leq 1-\left(\frac{\pi^{2}}{4}-1\right) \delta=z(0) \leq z(t), \quad \text { and } \\
& z(t) \leq z\left(\frac{\pi}{2}\right)=1 .
\end{aligned}
$$

Let $P \in \mathbf{R}^{1}$ and $t_{0} \in\left[-\sin ^{-1}(k / b), \sin ^{-1}(1 / b)\right]$ such that

$$
P=\max _{t \in\left[-\sin ^{-1}(k / b), \sin ^{-1}(1 / b)\right]}(Z(t)-z(t))=Z\left(t_{0}\right)-z\left(t_{0}\right) .
$$

Thus

$$
Z(t) \leq z(t)+P \quad \text { for } t \in\left[-\sin ^{-1}(k / b), \sin ^{-1}(1 / b)\right] \quad \text { and } \quad Z\left(t_{0}\right)=z\left(t_{0}\right)+P .
$$

Suppose that $P>0$. Then $z+P$ satisfies the conditions in Theorem 2.3. (2.12) implies

$$
\begin{aligned}
& z\left(t_{0}\right)+P=Z\left(t_{0}\right) \\
& \leq \frac{1}{2}(z+P)^{\prime \prime}\left(t_{0}\right) \cos ^{2} t_{0}-(z+P)^{\prime}\left(t_{0}\right) \cos t_{0} \sin t_{0}+1-2 \delta \cos ^{2} t_{0} \\
& =\frac{1}{2} z^{\prime \prime}\left(t_{0}\right) \cos ^{2} t_{0}-z^{\prime}\left(t_{0}\right) \cos t_{0} \sin t_{0}+1-2 \delta \cos ^{2} t_{0} \\
& =z\left(t_{0}\right) .
\end{aligned}
$$

This contradicts the assumption $P>0$. Thus $P \leq 0$ and $(3.2$ holds. That means

$$
\sqrt{\lambda} \geq \frac{|\nabla t|}{\sqrt{z(t)}}
$$

Note that the eigenfunction $v$ of the first nonzero eigenvalue has exactly two nodal domains $D^{+}=\{x: v(x)>0\}$ and $D^{-}=\{x: v(x)<0\}$ and that the nodal set $v^{-1}(0)$ is compact (see [1] and [2]). Take $q_{1}$ on $M$ such that $v\left(q_{1}\right)=1=\sup _{M} v$ and $q_{2} \in v^{-1}(0)$ such that distance $d\left(q_{1}, q_{2}\right)=$ distance $d\left(q_{1}, v^{-1}(0)\right)$. Let $L$ be the minimum geodesic segment joining $q_{1}$ and $q_{2}$. We integrate both sides of (3.7) along $L$ and change variable, and let $b \rightarrow 1$. Let $r_{+}, r_{-}$be the radius of the largest interior ball in $D^{+}, D^{-}$respectively, that is,

$$
r_{+}=\max _{x \in D_{+}} \operatorname{dist}\left(x, v^{-1}(0)\right)
$$

and

$$
r_{-}=\max _{x \in D_{-}} \operatorname{dist}\left(x, v^{-1}(0)\right) .
$$

Set $r=\max \left\{r_{+}, r_{-}\right\}$. Then

$$
r \sqrt{\lambda} \geq \int_{L} \frac{|\nabla t|}{\sqrt{z(t)}} d l=\int_{0}^{\frac{\pi}{2}} \frac{1}{\sqrt{z(t)}} d t \geq \frac{\left(\int_{0}^{\pi / 2} d t\right)^{\frac{3}{2}}}{\left(\int_{0}^{\pi / 2} z(t) d t\right)^{\frac{1}{2}}} \geq\left(\frac{\left(\frac{\pi}{2}\right)^{3}}{\int_{0}^{\pi / 2} z(t) d t}\right)^{\frac{1}{2}} .
$$

Square the two sides. Then

$$
\lambda \geq \frac{\pi^{3}}{8 r^{2} \int_{0}^{\pi / 2} z(t) d t}
$$


Now

$$
\int_{0}^{\frac{\pi}{2}} z(t) d t=\int_{0}^{\frac{\pi}{2}}[1+\delta \xi(t)] d t=\frac{\pi}{2}(1-\delta),
$$

by (3.12) in Lemma 3.1. That is,

$$
\lambda \geq \frac{\pi^{2}}{4 r^{2}(1-\delta)} \quad \text { and } \quad \lambda \geq \frac{1}{2}(n-1) K+\frac{\pi^{2}}{4 r^{2}} .
$$

This completes the proof.

We now present a lemma that is used in the proof of Theorem 1.1

Lemma 3.1. Let

$$
\xi(t)=\frac{\cos ^{2} t+2 t \sin t \cos t+t^{2}-\frac{\pi^{2}}{4}}{\cos ^{2} t} \quad \text { on }\left[-\frac{\pi}{2}, \frac{\pi}{2}\right] .
$$

Then the function $\xi$ satisfies the following:

$$
\begin{aligned}
& \frac{1}{2} \xi^{\prime \prime} \cos ^{2} t-\xi^{\prime} \cos t \sin t-\xi=2 \cos ^{2} t \quad \text { in }\left(-\frac{\pi}{2}, \frac{\pi}{2}\right), \\
& \xi^{\prime} \cos t-2 \xi \sin t=4 t \cos t, \\
& \int_{0}^{\frac{\pi}{2}} \xi(t) d t=-\frac{\pi}{2}, \\
& 1-\frac{\pi^{2}}{4}=\xi(0) \leq \xi(t) \leq \xi\left( \pm \frac{\pi}{2}\right)=0 \quad \text { on }\left[-\frac{\pi}{2}, \frac{\pi}{2}\right], \\
& \xi^{\prime} \text { is increasing on }\left[-\frac{\pi}{2}, \frac{\pi}{2}\right] \text { and } \xi^{\prime}\left( \pm \frac{\pi}{2}\right)= \pm \frac{2 \pi}{3}, \\
& \xi^{\prime}(t)<0 \text { on }\left(-\frac{\pi}{2}, 0\right) \text { and } \xi^{\prime}(t)>0 \text { on }\left(0, \frac{\pi}{2}\right), \\
& \xi^{\prime \prime}\left( \pm \frac{\pi}{2}\right)=2, \xi^{\prime \prime}(0)=2\left(3-\frac{\pi^{2}}{4}\right) \text { and } \xi^{\prime \prime}(t)>0 \text { on }\left[-\frac{\pi}{2}, \frac{\pi}{2}\right], \\
& \left(\frac{\xi^{\prime}(t)}{t}\right)^{\prime}>0 \text { on }(0, \pi / 2) \text { and } 2\left(3-\frac{\pi^{2}}{4}\right) \leq \frac{\xi^{\prime}(t)}{t} \leq \frac{4}{3} \text { on }\left[-\frac{\pi}{2}, \frac{\pi}{2}\right], \\
& \xi^{\prime \prime \prime}\left(\frac{\pi}{2}\right)=\frac{8 \pi}{15}, \xi^{\prime \prime \prime}(t)<0 \text { on }\left(-\frac{\pi}{2}, 0\right) \text { and } \xi^{\prime \prime \prime}(t)>0 \text { on }\left(0, \frac{\pi}{2}\right) .
\end{aligned}
$$

Proof. For convenience, let $q(t)=\xi^{\prime}(t)$, i.e.,

$$
q(t)=\xi^{\prime}(t)=\frac{2\left(2 t \cos t+t^{2} \sin t+\cos ^{2} t \sin t-\frac{\pi^{2}}{4} \sin t\right)}{\cos ^{3} t} .
$$

Equation (3.10) and the values $\xi\left( \pm \frac{\pi}{2}\right)=0, \xi(0)=1-\frac{\pi^{2}}{4}$ and $\xi^{\prime}\left( \pm \frac{\pi}{2}\right)= \pm \frac{2 \pi}{3}$ can be verified directly from (3.9) and (3.13). The values of $\xi^{\prime \prime}$ at 0 and $\pm \frac{\pi}{2}$ can be computed via (3.10). By (3.11), $\left(\xi(t) \cos ^{2} t\right)^{\prime}=4 t \cos ^{2} t$. Therefore $\xi(t) \cos ^{2} t=\int_{\frac{\pi}{2}}^{t} 4 s \cos ^{2} s d s$, and

$$
\begin{aligned}
& \int_{-\frac{\pi}{2}}^{\frac{\pi}{2}} \xi(t) d t=2 \int_{0}^{\frac{\pi}{2}} \xi(t) d t=-8 \int_{0}^{\frac{\pi}{2}}\left(\frac{1}{\cos ^{2}(t)} \int_{t}^{\frac{\pi}{2}} s \cos ^{2} s d s\right) d t \\
= & -8 \int_{0}^{\frac{\pi}{2}}\left(\int_{0}^{s} \frac{1}{\cos ^{2}(t)} d t\right) s \cos ^{2} s d s=-8 \int_{0}^{\frac{\pi}{2}} s \cos s \sin s d s=-\pi .
\end{aligned}
$$


It is easy to see that $q$ and $q^{\prime}$ satisfy the following equations:

$$
\frac{1}{2} q^{\prime \prime} \cos t-2 q^{\prime} \sin t-2 q \cos t=-4 \sin t
$$

and

$$
\frac{\cos ^{2} t}{2\left(1+\cos ^{2} t\right)}\left(q^{\prime}\right)^{\prime \prime}-\frac{2 \cos t \sin t}{1+\cos ^{2} t}\left(q^{\prime}\right)^{\prime}-2\left(q^{\prime}\right)=-\frac{4}{1+\cos ^{2} t} .
$$

The last equation implies that $q^{\prime}=\xi^{\prime \prime}$ cannot achieve its nonpositive local minimum at a point in $\left(-\frac{\pi}{2}, \frac{\pi}{2}\right)$. On the other hand, $\xi^{\prime \prime}\left( \pm \frac{\pi}{2}\right)=2$, by equation (3.10), $\xi\left( \pm \frac{\pi}{2}\right)=$ 0 and $\xi^{\prime}\left( \pm \frac{\pi}{2}\right)= \pm \frac{2 \pi}{3}$. Therefore $\xi^{\prime \prime}(t)>0$ on $\left[-\frac{\pi}{2}, \frac{\pi}{2}\right]$ and $\xi^{\prime}$ is increasing. Since $\xi^{\prime}(t)=0$, we have $\xi^{\prime}(t)<0$ on $\left(-\frac{\pi}{2}, 0\right)$ and $\xi^{\prime}(t)>0$ on $\left(0, \frac{\pi}{2}\right)$. Similarly, from the equation

$$
\begin{gathered}
\frac{\cos ^{2} t}{2\left(1+\cos ^{2} t\right)}\left(q^{\prime \prime}\right)^{\prime \prime}-\frac{\cos t \sin t\left(3+2 \cos ^{2} t\right)}{\left(1+\cos ^{2} t\right)^{2}}\left(q^{\prime \prime}\right)^{\prime}-\frac{2\left(5 \cos ^{2} t+\cos ^{4} t\right)}{\left(1+\cos ^{2} t\right)^{2}}\left(q^{\prime \prime}\right) \\
=-\frac{8 \cos t \sin t}{\left(1+\cos ^{2} t\right)^{2}}
\end{gathered}
$$

we get the results in the last line of the lemma.

Set $h(t)=\xi^{\prime \prime}(t) t-\xi^{\prime}(t)$. Then $h(0)=0$ and $h^{\prime}(t)=\xi^{\prime \prime \prime}(t) t>0$ in $\left(0, \frac{\pi}{2}\right)$. Therefore $\left(\frac{\xi^{\prime}(t)}{t}\right)^{\prime}=\frac{h(t)}{t^{2}}>0$ in $\left(0, \frac{\pi}{2}\right)$. Note that $\frac{\xi^{\prime}(-t)}{-t}=\frac{\xi^{\prime}(t)}{t},\left.\frac{\xi^{\prime}(t)}{t}\right|_{t=0}=\xi^{\prime \prime}(0)=$ $2\left(3-\frac{\pi^{2}}{4}\right)$ and $\left.\frac{\xi^{\prime}(t)}{t}\right|_{t=\pi / 2}=\frac{4}{3}$. This completes the proof of the lemma.

\section{REFERENCES}

[1] I. Chavel, Eigenvalues in Riemannian Geometry, Academic Press, Orlando, Fl., 1984. MR0768584 (86g:58140)

[2] R. Courant and D. Hilbert, Methods of Mathematical Physics, volume I. Interscience, New York, 1953. MR0065391(16:426a)

[3] P. Li, Lecture Notes on Geometric Analysis, Lecture Notes Series, \#6, Seoul National University, Seoul, Korea. MR1320504 (96m:58269)

[4] P. Li and S. T. Yau, On the Schrödinger equation and the eigenvalue problem, Comm. Math. Phys, 88(1983), 309-318. MR0701919 (84k:58225)

[5] P. Li and S. T. Yau, Estimates of eigenvalues of a compact Riemannian manifold, AMS Proc. Symp. Pure Math., 36(1980), 205-239. MR0573435 (81i:58050)

[6] A. Lichnerowicz, Geometrie des groupes de transformations, Dunod, Paris, 1958. MR0124009 (23:A1329)

[7] Jun Ling, A lower bound for the gap between the first two eigenvalues of Schrödinger operators on convex domains in $\mathrm{S}^{n}$ or $\mathrm{R}^{n}$, Michigan Math. J., 40(1993), 259-270. MR1226831 (94h:35185)

[8] Jun Ling, A bound for the first fundamental gap. Ph.D. Dissertation, State University of New York at Buffalo.

[9] Jun Ling, Estimates on the lower bound of the first gap, Preprint, 2004.

[10] R. Schoen, and S. T. Yau, Lecture Notes on Differential Geometry, Conference Procedings and Lecture Notes in Geometry and Topology, Vol. 1, International Press, 1994. MR:1333601 (97d:53001)

[11] D. Yang, Lower bound estimates on the first eigenvalue for compact manifolds with positive Ricci curvature, Pacific Journal of Mathematics, 190(1999), 383-398. MR1722898 (2001b:53039)

[12] J.-Q. Zhong and H. C. Yang, On the estimate of the first eigenvalue of a compact Riemannian manifold, Sci. Sinica, Ser. A 27, (1984), 1265-1273. MR0794292 (87a:58162)

Department of Mathematics, Utah Valley State College, Orem, Utah 84058

E-mail address: lingju@uvsc.edu 